\title{
Periodic orbits in the case of a zero eigenvalue
}

\author{
Petre Birtea, Mircea Puta, Razvan Micu Tudoran
}

\begin{abstract}
We will show that if a dynamical system has enough constants of motion then a Moser-Weinstein type theorem can be applied for proving the existence of periodic orbits in the case when the linearized system is degenerate.
\end{abstract}

\section{Introduction.}

Finding periodic solutions of a system of ordinary differential equations is an old problem in mathematical physics going back to Lyapunov and Poincare. Periodic solutions were discovered first for linear conservative systems that appears in mechanics. The passage from linear to nonlinear systems was taken by Lyapunov [2] under the assumption of existence of an integral of motion and a certain nonresonance condition.

In 1973, Weinstein [4] proved that in the case of a Hamiltonian system with a positive definite Hamiltonian function the nonresonance condition is not necessary. Later, Moser [3] extended Weinstein's result to the case of a general dynamical system which posses a constant of motion. More precisely, let

$$
\dot{x}=X(x),
$$

be a dynamical system generated by the $C^{1}$ vector field $X$ on a differentiable manifold $M$ with $x_{0}$ an equilibrium point, i.e., $X\left(x_{0}\right)=0$. Consider the linearized equations for the equilibrium point $x_{0}$,

$$
\dot{z}=D X\left(x_{0}\right) \cdot z .
$$

Then we have the following result due to Moser [3].

Theorem (Moser) Let $I \in C^{2}$ be an integral of motion for (1.1) with $d I\left(x_{0}\right)=0$. If

(i) $D X\left(x_{0}\right)$ is a non-singular matrix,

(ii) $D X\left(x_{0}\right)$ has a pair of pure complex eigenvalues $\pm i \omega$ with $\omega \neq 0$,

(iii) $d^{2} I\left(x_{0}\right)$ is positive definite,

then for sufficiently small $\epsilon$ any integral surface

$$
I(x)=I\left(x_{0}\right)+\varepsilon^{2}
$$


contains at least one periodic solution of $X$ whose period is close to the period of the corresponding linear system around $x_{0}$.

The condition $(i)$ of the above theorem implies that the linearized system around the critical point $x_{0}$ can not have a zero eigenvalue. This restriction makes the theorem unapplicable to a series of examples. We will show that in the case when for (1.1) one can find enough constants of motion a similar result can be applied for proving the existence of periodic orbits. We will also illustrate this with two examples.

\section{The main result.}

Theorem 2.1. Let $\dot{x}=X(x)$ be a dynamical system, $x_{0}$ an equilibrium point, i.e., $X\left(x_{0}\right)=0$ and $C:=\left(C_{1}, \ldots, C_{k}\right): M \rightarrow \mathbb{R}^{k}$ a vector valued constant of motion for the above dynamical system with $C\left(x_{0}\right)$ a regular value for $C$. If

(i) the eigenspace corresponding to the eigenvalue zero of the linearized system around $x_{0}$ has dimension $k$,

(ii) $D X\left(x_{0}\right)$ has a pair of pure complex eigenvalues $\pm i \omega$ with $\omega \neq 0$,

(iii) there exist a constant of motion $I: M \rightarrow \mathbb{R}$ for the vector field $X$ with $d I\left(x_{0}\right)=0$ and such that

$$
d^{2} I\left(x_{0}\right)_{\left.\right|_{W \times W}}>0
$$

where $W=\bigcap_{i=1}^{k} \operatorname{ker} d C_{i}\left(x_{0}\right)$,

then for each sufficiently small $\varepsilon \in \mathbb{R}$, any integral surface

$$
I(x)=I\left(x_{0}\right)+\varepsilon^{2}
$$

contains at least one periodic solution of $X$ whose period is close to the period of the corresponding linear system around $x_{0}$.

Proof. If $C_{i} \in C^{\infty}(M, \mathbb{R})$ is a constant of motion for the dynamic generated by the vector field $X$ then $D X\left(x_{0}\right) \nabla C_{i}\left(x_{0}\right)=0$, and hence $\nabla C_{i}\left(x_{0}\right) \in \operatorname{ker} D X\left(x_{0}\right)$.

Because $C\left(x_{0}\right)$ is a regular value for $C$ we have that $d C_{i}\left(x_{0}\right), i=\overline{1, k}$ are linearly independent vectors in the tangent space $T_{x_{0}} M$. Then, hypothesis $(i)$ and the fact that $C_{1}, \ldots, C_{k} \in C^{\infty}(M, \mathbb{R})$ are constants of motion for $X$ implies the following equality,

$$
\operatorname{span}\left\{\nabla C_{i}\left(x_{0}\right): i=\overline{1, k}\right\}=\operatorname{ker} D X\left(x_{0}\right)\left(=V_{\lambda=0}\right)
$$

where $V_{\lambda=0}$ is the eigenspace corresponding to the zero eigenvalue of the matrix which is canonically associated to the linear part at the equilibrium of interest $x_{0}$ of our system determined by $X$.

This argument implies that the reduced system

$$
\left\{\begin{array}{l}
\dot{x}=X(x) \\
C(x)=C\left(x_{0}\right)
\end{array}\right.
$$


which is the original system restricted to the submanifold $C^{-1}\left(C\left(x_{0}\right)\right)$ has the linearization about $x_{0}$ without eigenvalue zero.

The function $I_{\mid C^{-1}\left(C\left(x_{0}\right)\right)}: C^{-1}\left(C\left(x_{0}\right)\right) \rightarrow \mathbb{R}$ is a first integral for the reduced system with $d\left(I_{\mid C^{-1}\left(C\left(x_{0}\right)\right)}\right)\left(x_{0}\right)=0$ and hypothesis $(i i i)$ obviously implies that $d^{2}\left(I_{\mid C^{-1}\left(C\left(x_{0}\right)\right)}\right)\left(x_{0}\right)>$ 0 . By the Moser theorem we have that for sufficiently small $\varepsilon \in \mathbb{R}$, any integral surface

$$
I(x)=I\left(x_{0}\right)+\varepsilon^{2}
$$

contains at least one periodic solution of the reduced system and hence of the initial system.

Remark 2.1. If the dynamic (1.1) is Hamilton-Poisson and $x_{0}$ is regular point in the sense that it is contained in a maximal dimension symplectic leaf of $(M,\{\})$ which is determined by the Casimirs $C_{1}, \ldots, C_{k}$, then by the theorem of Weinstein [4] one has the existence of $(\operatorname{dim} P-k) / 2$ periodic orbits.

\section{Examples.}

Rigid body with one control. Let us consider the rigid body dynamics with one control,

$$
\left\{\begin{array}{l}
\dot{m}_{1}=a_{1} m_{2} m_{3} \\
\dot{m}_{2}=a_{2} m_{1} m_{3} \\
\dot{m}_{3}=\left(a_{3}-l\right) m_{1} m_{2}
\end{array}\right.
$$

where $l \in \mathbb{R}$ is the gain parameter.

Let us make now the following notation $\alpha:=\frac{a_{3}-l}{a_{3}}$. Then it is not hard to see that our dynamics (3.1) has the following Hamilton-Poisson realization $\left(\mathbb{R}^{3}, \Pi_{\alpha}, H_{\alpha}\right)$, where

$$
\Pi_{\alpha} \stackrel{\text { def }}{=}\left[\begin{array}{rrr}
0 & -m_{3} & \alpha m_{2} \\
m_{3} & 0 & -\alpha m_{1} \\
-\alpha m_{2} & \alpha m_{1} & 0
\end{array}\right]
$$

is the Poisson structure and $H_{\alpha}\left(m_{1}, m_{2}, m_{3}\right) \stackrel{\text { def }}{=} \frac{1}{2}\left(\frac{m_{1}^{2}}{I_{1}}+\frac{m_{2}^{2}}{I_{2}}+\frac{m_{3}^{2}}{\alpha I_{3}}\right)$ is the Hamiltonian function. Moreover, the smooth function $C_{\alpha} \in C^{\infty}\left(\mathbb{R}^{3}, \mathbb{R}\right)$ given by

$$
C_{\alpha}\left(m_{1}, m_{2}, m_{3}\right) \stackrel{\text { def }}{=} \alpha m_{1}^{2}+\alpha m_{2}^{2}+m_{3}^{2}
$$

is a Casimir of our Poisson configuration $\left(\mathbb{R}^{3}, \Pi_{\alpha}\right)$.

Let us concentrate now to the equilibrium state

$$
e_{1}^{M}=(M, 0,0), M \in \mathbb{R}^{*}
$$

of our dynamics (3.1). Then under the restriction $l<a_{3}$ we have succesivelly, 
(i) The restriction of the dynamics (3.1) to the coadjoint orbit

$$
\alpha m_{1}^{2}+\alpha m_{2}^{2}+m_{3}^{2}=\alpha M^{2}
$$

gives rise to a Hamiltonian system on a symplectic manifold.

(ii) $\operatorname{span}\left(\nabla C_{\alpha}\left(e_{1}^{M}\right)\right)=V_{\lambda=0}=\operatorname{span}\left(\left[\begin{array}{l}1 \\ 0 \\ 0\end{array}\right]\right)$ where

$$
V_{\lambda=0}=\left\{\left[\begin{array}{l}
m_{1} \\
m_{2} \\
m_{3}
\end{array}\right] \in \mathbb{R}^{3} \mid A\left(e_{1}^{M}\right)\left[\begin{array}{l}
m_{1} \\
m_{2} \\
m_{3}
\end{array}\right]=\left[\begin{array}{l}
0 \\
0 \\
0
\end{array}\right]\right\}
$$

$A\left(e_{1}^{M}\right)$ being the matrix of the linear part of the dynamics (3.1) at the equilibrium of interest $e_{1}^{M}, M \in \mathbb{R}^{*}$.

(iii) The matrix of the linear part of our reduced dynamics to (3.2) has at the equilibrium $e_{1}^{M}$ the following characteristic roots:

$$
\lambda_{1,2}= \pm M i \sqrt{-a_{2}\left(a_{3}-l\right)}
$$

(iv) The smooth function $F_{\frac{1}{\alpha I_{1}}} \in C^{\infty}\left(\mathbb{R}^{3}, \mathbb{R}\right)$ given by:

$$
F_{\frac{1}{\alpha I_{1}}}\left(m_{1}, m_{2}, m_{3}\right)=\frac{1}{2}\left(\frac{m_{1}^{2}}{I_{1}}+\frac{m_{2}^{2}}{I_{2}}+\frac{m_{3}^{2}}{\alpha I_{3}}\right)-\frac{1}{2 \alpha I_{1}}\left(\alpha m_{1}^{2}+\alpha m_{2}^{2}+m_{3}^{2}\right)
$$

is a constant of motion and $e_{1}^{M}$ is a local minimum of $F_{\frac{1}{a l I_{1}}}$ with the constraint (3.2).

Then via Theorem 2.1 we have:

Proposition 3.1. If $l<a_{3}$ then the reduced dynamics to the coadjoint orbit (3.2) has near the equilibrium state $e_{1}^{M}, M \in \mathbb{R}^{*}$ at least one periodic solution whose period is close to

$$
\frac{2 \pi}{|M| \sqrt{-a_{2}\left(a_{3}-l\right)}} \text {. }
$$

Remark 3.1. Similar results can be also obtained for the equilibrium states

$$
e_{2}^{M}=(0, M, 0), M \in \mathbb{R}^{*}
$$

and

$$
e_{3}^{M}=(0,0, M), M \in \mathbb{R}^{*}
$$


Clebsch system. It is well known that the Clebsch system can be written in the following form:

$$
\left\{\begin{array}{l}
\dot{x}_{1}=x_{2} p_{3}-x_{3} p_{2} \\
\dot{x}_{2}=x_{3} p_{1}-x_{1} p_{3} \\
\dot{x}_{3}=x_{1} p_{2}-x_{2} p_{1} \\
\dot{p}_{1}=\left(a_{3}-a_{2}\right) x_{2} x_{3} \\
\dot{p}_{2}=\left(a_{1}-a_{3}\right) x_{1} x_{3} \\
\dot{p}_{3}=\left(a_{2}-a_{1}\right) x_{1} x_{2}
\end{array}\right.
$$

where

$$
\begin{aligned}
& a_{1}, a_{2}, a_{3} \in \mathbb{R} \\
& a_{1}>0, a_{2}>0, a_{3}>0 \\
& a_{1} \neq a_{2} \neq a_{3}
\end{aligned}
$$

(see for details Dubrovin, Krichever and Novikov [1]).

It is not hard to see that the smooth functions $H, C, D \in C^{\infty}\left(\mathbb{R}^{6}, \mathbb{R}\right)$ given by:

$$
\begin{aligned}
& H\left(x_{1}, x_{2}, x_{3}, p_{1}, p_{2}, p_{3}\right)=\frac{1}{2}\left(a_{1} x_{1}^{2}+a_{2} x_{2}^{2}+a_{3} x_{3}^{2}+p_{1}^{2}+p_{2}^{2}+p_{3}^{2}\right) \\
& C\left(x_{1}, x_{2}, x_{3}, p_{1}, p_{2}, p_{3}\right)=\frac{1}{2}\left(x_{1}^{2}+x_{2}^{2}+x_{3}^{2}\right) \\
& D\left(x_{1}, x_{2}, x_{3}, p_{1}, p_{2}, p_{3}\right)=x_{1} p_{1}+x_{2} p_{2}+x_{3} p_{3}
\end{aligned}
$$

are constants of motion for the Clebsch system.

Let us concentrate now to the equilibrium state $e_{1}^{M}=(M, 0,0,0,0,0), M \in \mathbb{R}^{*}$. Then under the restrictions:

$$
a_{3}>a_{1} \text { and } a_{2}>a_{1}
$$

we have successively,

(i) $\operatorname{span}\left(\nabla C_{\alpha}\left(e_{1}^{M}\right), \nabla D\left(e_{1}^{M}\right)\right)=V_{\lambda=0}$ where

$$
V_{\lambda=0}=\left\{\left[\begin{array}{c}
m_{1} \\
m_{2} \\
m_{3} \\
p_{1} \\
p_{2} \\
p_{3}
\end{array}\right] \in \mathbb{R}^{6} \mid A\left(e_{1}^{M}\right)\left[\begin{array}{c}
m_{1} \\
m_{2} \\
m_{3} \\
p_{1} \\
p_{2} \\
p_{3}
\end{array}\right]=\left[\begin{array}{l}
0 \\
0 \\
0 \\
0 \\
0 \\
0
\end{array}\right]\right\},
$$

$A\left(e_{1}^{M}\right)$ being the matrix of the linear part of the dynamics (3.3) at the equilibrium $e_{1}^{M}$.

(ii) The matrix of the linear part of our reduced dynamics to the constraint

$$
\left\{\begin{array}{l|l}
\left(x_{1}, x_{2}, x_{3}, p_{1}, p_{2}, p_{3}\right) \in \mathbb{R}^{6} & \begin{array}{l}
x_{1}^{2}+x_{2}^{2}+x_{3}^{2}=M^{2} \\
x_{1} p_{1}+x_{2} p_{2}+x_{3} p_{3}=0
\end{array}
\end{array}\right\},
$$

at the equilibrium $e_{1}^{M}$ has the following characteristic roots:

$$
\begin{aligned}
& \lambda_{1,2}= \pm i M \sqrt{a_{3}-a_{1}}, \\
& \lambda_{3,4}= \pm i M \sqrt{a_{2}-a_{1}} .
\end{aligned}
$$


(iii) The smooth function $F_{a_{1}} \in C^{\infty}\left(\mathbb{R}^{6}, \mathbb{R}\right)$ given by:

$$
\begin{aligned}
F_{a_{1}}\left(x_{1}, x_{2}, x_{3}, p_{1}, p_{2}, p_{3}\right) & =\frac{1}{2}\left(a_{1} x_{1}^{2}+a_{2} x_{2}^{2}+a_{3} x_{3}^{2}+p_{1}^{2}+p_{2}^{2}+p_{3}^{2}\right) \\
& -\frac{a_{1}}{2}\left(x_{1}^{2}+x_{2}^{2}+x_{3}^{2}\right)
\end{aligned}
$$

is a constant of motion and $e_{1}^{M}$ is a local minimum of $F_{a_{1}}$ with the constraint (3.4).

Then via Theorem 2.1 we have:

Proposition 3.2. If $a_{2}<a_{1}$ and $a_{3}>a_{1}$ then the reduced dynamics to (3.4) has near $e_{1}^{M}, M \in \mathbb{R}^{*}$ at least one periodic solution whose period is close to $\frac{2 \pi}{|M| \sqrt{a_{3}-a_{1}}}$ and $\frac{2 \pi}{|M| \sqrt{a_{2}-a_{1}}}$.

Remark 3.2. Similar results can be also obtained for the equilibrium states:

$$
e_{2}^{M}=(0, M, 0,0,0,0), M \in \mathbb{R}^{*}
$$

and

$$
e_{3}^{M}=(0,0, M, 0,0,0), M \in \mathbb{R}^{*}
$$

\section{References}

[1] Dubrovin, B., Krichever, I., Novikov, S., Integrable systems, in Encyclopedia of Math. Sci., Springer Verlag, Berlin, 4 (1990), pp. 173-280.

[2] Lyapunov, M. A., Problème général de la stabilité du mouvement, Ann. Fac. Sci., Touluse 2 (1907), pp. 203-474.

[3] Moser, J., Periodic orbits and a theorem by Alan Weinstein, Communications Pure Appl. Math., 29 (1976), pp. 727-747.

[4] Weinstein, A., Normal modes for non-linear Hamilronian systems, Inv. Math., 20 (1973), pp. 47-57. 